NCUSF 3

Jan 2010

\title{
Factoring the Dispersion Relation in the Presence of Lorentz Violation
}

\author{
Don Colladay, Patrick McDonald, and David Mullins \\ New College of Florida \\ Sarasota, FL, 34243, U.S.A.
}

\begin{abstract}
We produce an explicit formula for the dispersion relation for the Dirac Equation in the Standard Model Extension (SME) in the presence of Lorentz violation. Our expression is obtained using a novel techniques which exploit the algebra of quaternions. The dispersion relation is found to conveniently factor in two special cases that each involve a mutually exclusive set of non-vanishing Lorentz-violating parameters. This suggests that a useful approach to studies of Lorentz-violating models is to split the parameter space into two separate pieces, each of which yields a simple, tractable dispersion relation that can be used for analysis.
\end{abstract}

Submitted for Publication in JMP 


\section{INTRODUCTION}

As a low energy limit of a fundamental theory, the Standard Model (SM) has proven a remarkable success. As a result, well-known properties of the model, in particular, Lorentz invariance, have become default characteristics built in to many models which purport to underlie the SM at higher energy scales. While experimental evidence indicates that in presently accessible energy regimes violation of Lorentz symmetry must be small, there is no a priori necessity for insisting that Lorentz invariance hold for theories whose intent is to probe physics at higher energy. Indeed, Lorentz violation may serve as a signal for new physics at the Plank scale [1].

Motivated by these and related observations, a model has been constructed which facilitates the study of the possible effects of incorporating Lorentz violation in the SM. This model, called the Standard Model Extension (SME), has been studied extensively $[2,3]$. The SME framework exhibits many of the properties of standard quantum field theories including gauge invariance, energy-momentum conservation, causality and stability (in concordant frames)[4], observer Lorentz invariance and hermiticity. In addition, numerous renormalizability properties of the theory have been established [5, 6, 7], etc, and various implications for particle theory, gravity (Lorentz violation provides an alternative means of generating theories of gravity [8]) and cosmology (see [9] for a study of the relationship of Lorentz violation to cosmic microwave background data) have been discussed.

In addition to the theoretical work done on the SME, numerous experiments have been performed to bound the LV effects predicted by the theory. These experiments involve numerous aspects of the SM (bounds associated to electrons, photons, neutrinos, and hadrons, etc) An exhaustive list and a brief discussion of relevant experimental results are contained in a well-maintained set of data tables [10].

Of central importance in the design, implementation and interpretation of any experiment intended to probe Lorentz violation is a precise understanding of the associated dispersion relation. The goal of this paper is to give a detailed description of the dispersion relation for the Dirac operator associated to the free fermion sector of the SME. In carrying out our goal we discover some new properties of the dispersion 
relation. In particular, our analysis provides a natural splitting of the parameters determining Lorentz violation into two disjoint sets. This partition includes as special cases all previously investigated special cases in which a precise description of the dispersion relation is easy to establish. In addition, our analysis provides new results for the special case in which Lorentz violation is coupled to spin. While we offer no explanation for the emergence of our partition as a manifestation of the properties of the SME, the possibility that hidden symmetries might be their source provides an intriguing problem for future investigation.

This paper is organized as follows: In the second section we provide a introduction to the SME in which we establish both notation and basic defining features of the model. In the next section we use a representation involving the quaternions to investigate the Dirac equation and associated dispersion relation for the SME. In this section we also establish our fundamental result: an explicit polynomial representation for the dispersion relation in terms of the Lorentz violating parameters which define the theory. In the fourth section we discuss consequences of our formula, including consistency checks with existing literature, a complete analysis of several important special cases, and the discovery of new relationships between Lorentz violating parameters. In the final section of the paper we discuss our conclusions and some potential directions for future work.

\section{NOTATION, CONVENTIONS AND BACKGROUND}

Let $\sigma_{j}$ be the Pauli matrices, $\gamma_{\mu}$ denote the standard gamma matrices, and $\eta_{\lambda \mu}=$ $\operatorname{diag}(1,-1,-1,-1)$ the Minkowski metric with signature -2 . Thus,

$$
i \sigma_{1}=\left(\begin{array}{cc}
0 & i \\
i & 0
\end{array}\right) \quad i \sigma_{2}=\left(\begin{array}{cc}
0 & 1 \\
-1 & 0
\end{array}\right) \quad i \sigma_{3}=\left(\begin{array}{cc}
i & 0 \\
0 & -i
\end{array}\right)
$$

and

$$
\gamma^{0}=\left(\begin{array}{cc}
I & 0 \\
0 & -I
\end{array}\right) \quad \gamma^{j}=\left(\begin{array}{cc}
0 & \sigma_{j} \\
-\sigma_{j} & 0
\end{array}\right)
$$

where $i$ is the imaginary unit. The SME Lorentz violating Lagrangian for a single spin- $\frac{1}{2}$ fermion is given by

$$
\mathcal{L}=i \bar{\psi} \Gamma^{\nu} \partial_{\nu} \psi-\bar{\psi} M \psi
$$


where

$$
\begin{aligned}
\Gamma^{\nu} & =\gamma^{\nu}+c^{\mu \nu} \gamma_{\mu}+d^{\mu \nu} \gamma_{5} \gamma_{\mu}+e^{\nu}+i f^{\nu} \gamma_{5}+\frac{1}{2} g^{\lambda \mu \nu} \sigma_{\lambda \mu} \\
M & =m+a_{\mu} \gamma^{\mu}+b_{\mu} \gamma_{5} \gamma^{\mu}+\frac{1}{2} H_{\mu \nu} \sigma^{\mu \nu}
\end{aligned}
$$

The coefficients $c_{\mu \nu}, d_{\mu \nu}, e_{\nu}, f_{\nu}, g_{\lambda \mu \nu}, a_{\mu}, b_{\mu}, H_{\mu \nu}$ governing Lorentz violation are assumed small. Since the Lagrangian is Hermitian, the parameters are real. In addition, the parameters $c_{\nu \mu}$ and $d_{\nu \mu}$ can be taken to be traceless, $H_{\mu \nu}$ antisymmetric, and $g^{\lambda \mu \nu}$ antisymmetric in the first two components. The parameters $a_{\mu}, b_{\mu}, H_{\mu \nu}$, have the dimension of mass, while the remaining parameters are dimensionless.

As mentioned above, the SME exhibits many of the properties of standard quantum field theories including gauge invariance, energy-momentum conservation, causality, stability, observer Lorentz invariance, hermiticity and power counting renormalizability. In addition, any theory that generates the SM and exhibits spontaneous Lorentz and CPT violation contains the SME as an appropriate limit [2].

The Dirac equation associated to the Lagrangian (3) is given by

$$
\left(i \Gamma^{\nu} \partial_{\nu}-M\right) \psi=0
$$

or, in momentum space coordinates (using $\psi(x)=e^{-i p \cdot x} u(p)$ for now)

$$
\left(\Gamma^{\nu} p_{\nu}-M\right) \psi=0
$$

The Dirac operator $\left(\Gamma^{\nu} p_{\nu}-M\right)$ is a $4 \times 4$ matrix with complex entries. The dispersion relation characterizes the null space of the Dirac operator and is given by

$$
\operatorname{det}\left(\Gamma^{\nu} p_{\nu}-M\right)=0 \text {. }
$$

Expression (8) describes the zeroes of a fourth order polynomial in $p^{0}$ whose coefficients depend smoothly on the Lorentz violating parameters and on the momentum vector $\vec{p}=\left(p^{1}, p^{2}, p^{3}\right)$. The explicit covariant form of this dispersion relation is presented in the literature [11], however, the general expression of the dispersion relation yields little insight into specific parameter choices that allow for a simple factorization. The present work remedies the situation by using new techniques to analyze the resulting algebraic varieties that arise from this dispersion relation. 
We will denote by $\mathcal{C}$ the charge conjugation operator, $\mathcal{P}$ the parity inversion operator, and $\mathcal{T}$ the time reversal operator. It is of interest to understand the effect of these discrete symmetries on the structure of the Lorentz-violating theory.

Using the Dirac representation we write the charge conjugation matrix as

$$
C=i \gamma^{2} \gamma^{0}=\left(\begin{array}{cc}
0 & -i \sigma_{2} \\
-i \sigma_{2} & 0
\end{array}\right)
$$

The C, P and T symmetries of the SM are given at the level of the Dirac operator by the transformations

$$
\begin{aligned}
& \mathcal{C}: \Gamma^{\nu} p_{\nu}-M \quad \longrightarrow C \gamma^{0}\left(\Gamma^{\nu} p_{\nu}-M\right)^{*} C \gamma^{0} \\
& \mathcal{P}: \Gamma^{\nu} p_{\nu}-M \longrightarrow \gamma^{0}\left(\Gamma^{\nu} p_{\nu}-M\right) \gamma^{0} \\
& \mathcal{T}: \Gamma^{\nu} p_{\nu}-M \longrightarrow i \gamma^{5} C\left(\Gamma^{\nu} p_{\nu}-M\right)^{*} i \gamma^{5} C .
\end{aligned}
$$

From (8) and basic invariant theory of the real valued determinant, it is clear that the dispersion relation is unaltered by any of the above transformations. This indicates that the generic effect of these discrete transformations is to permute the energy eigenstates in various ways. The effect of CPT on the SME Lagrangian can be neatly summarized for our purposes. For fixed $\vec{p}, b^{\mu}, d^{\mu \nu}, g^{\mu \nu \lambda}, H^{\mu \nu}$ we will denote by $R t\left(p^{0}\left(\vec{p}, b^{\mu}, d^{\mu \nu}, g^{\mu \nu \lambda} H^{\mu \nu}\right)\right)$ the roots of the fourth order polynomial in $p_{0}$ defined by (8). Then the above transformation properties of the Dirac operator yield the following root permutations:

$$
\begin{aligned}
& \mathcal{C}: R t\left(p^{0}\left(\vec{p}, b^{\mu}, d^{\mu \nu}, g^{\mu \nu \lambda}, H^{\mu \nu}\right)\right) \quad \longrightarrow \quad-R t\left(p^{0}\left(-\vec{p}, b^{\mu},-d^{\mu \nu}, g^{\mu \nu \lambda},-H^{\mu \nu}\right)\right) \\
& \mathcal{P}: R t\left(p^{0}\left(\vec{p}, b^{\mu}, d^{\mu \nu}, g^{\mu \nu \lambda}, H^{\mu \nu}\right)\right) \longrightarrow R t\left(p^{0}\left(-\vec{p},-b_{\mu},-d_{\mu \nu}, g_{\mu \nu \lambda}, H_{\mu \nu}\right)\right) \\
& \mathcal{T}: R t\left(p^{0}\left(\vec{p}, b^{\mu}, d^{\mu \nu}, g^{\mu \nu \lambda}, H^{\mu \nu}\right)\right) \longrightarrow R t\left(p^{0}\left(-\vec{p}, b_{\mu}, d_{\mu \nu},-g_{\mu \nu \lambda},-H_{\mu \nu}\right)\right)
\end{aligned}
$$

\section{DISPERSION RELATION USING QUATERNIONS}

To further analyze the dispersion relation (8) we employ a quaternion algebra. More precisely, let $1, \hat{i}, \hat{j}, \hat{k}$ denote the usual basis for the quaternions (denoted $\mathbf{H}$ ), and let $i$ denote the usual complex imaginary unit. Elements of $\mathbf{H}$ are expressions of 
the form $a+\hat{i} b+\hat{j} c+\hat{k} d$ where $a, b, c, d$ are real. Denote by a "bar" quaternionic conjugation:

$$
\overline{\hat{i}}=-\hat{i} \quad \overline{\hat{j}}=-\hat{j} \quad \overline{\hat{k}}=-\hat{k} \quad \bar{i}=i .
$$

We can identify quaternions with $2 \times 2$ complex matrices of the form $\left(\begin{array}{cc}z & w \\ -\bar{w} & \bar{z}\end{array}\right)$ :

$$
a+\hat{i} b+\hat{j} c+\hat{k} d \rightarrow\left(\begin{array}{cc}
a+i b & c+i d \\
-c+i d & a-i b
\end{array}\right)
$$

This identification is an isomorphism of skew fields; in particular multiplication of quaternions is mapped to matrix multiplication. Note that the map identifies the Pauli matrices with elements of the standard basis of the quaternions:

$$
i \sigma_{1} \longrightarrow \hat{k} \quad i \sigma_{2} \longrightarrow \hat{j} \quad i \sigma_{3} \longrightarrow \hat{i}
$$

We will refer to matrices of the form (14) as quaternionic matrices. These matrices play a central role in our approach ${ }^{1}$.

Quaternionic matrices can be used to represent general $2 \times 2$ complex matrices. More precisely, every $2 \times 2$ complex matrix has a unique representation of the form $A+i B$ where $A$ and $B$ are quaternionic. This representation extends inductively to higher dimension. In particular, given a general $4 \times 4$ complex matrix $M$, we can decompose $M$ into four $2 \times 2$ complex matrices. Since each complex $2 \times 2$ block has a unique representation of the form $A+i B$ with $A$ and $B$ quaternionic, $M$ has a unique decomposition of the form $M_{1}+i M_{2}$ where each $M_{i}$ is a $2 \times 2$ block matrix with each block given by a quaternionic matrix. Since each block is quaternionic, every $4 \times 4$ complex matrix has a unique representation of the form $Q_{1}+i Q_{2}$ where the $Q_{i}$ are $2 \times 2$ matrices with entries which are quaternions. Implementing this construction for the gamma matrices gives

$$
\gamma^{1} \longrightarrow i\left(\begin{array}{cc}
0 & -\hat{k} \\
\hat{k} & 0
\end{array}\right), \quad \gamma^{2} \longrightarrow i\left(\begin{array}{cc}
0 & -\hat{j} \\
\hat{j} & 0
\end{array}\right), \quad \gamma^{3} \longrightarrow i\left(\begin{array}{cc}
0 & -\hat{i} \\
\hat{i} & 0
\end{array}\right)
$$

and the standard Dirac equation becomes the $2 \times 2$ matrix equation

$$
\left[\left(\begin{array}{cc}
p_{0}-m & 0 \\
0 & -p_{0}-m
\end{array}\right)+i\left(\begin{array}{cc}
0 & \hat{p} \\
-\hat{p} & 0
\end{array}\right)\right]\left(\begin{array}{l}
\phi \\
\xi
\end{array}\right)=\left(\begin{array}{l}
0 \\
0
\end{array}\right)
$$

\footnotetext{
${ }^{1}$ Our approach should not be confused with attempts in the literature to reformulate quantum mechanics itself in terms of quaternions [12]. We simply use the algebraic properties of the quaternions as a computational tool in the conventional framework of complex-valued fields.
} 
where

$$
\hat{p}=i \vec{p} \cdot \vec{\sigma}=p_{3} \hat{i}+p_{2} \hat{j}+p_{1} \hat{k} .
$$

We will henceforth always use a "hat" to represent quaternions with no real component and standard script to denote quaternions with no non-real component. Note the reversal of first and third components of the imaginary quaternion relative to the standard three-vector notation. With this convention we can express the Dirac equation for the SME Lagrangian:

$$
\left[\left(\begin{array}{cc}
x & \alpha_{0}+\hat{\alpha} \\
-\alpha_{0}+\hat{\alpha} & -y
\end{array}\right)+i\left(\begin{array}{cc}
\hat{\epsilon} & \hat{p} \\
-\hat{p} & \hat{\delta}
\end{array}\right)\right]\left(\begin{array}{l}
\phi \\
\xi
\end{array}\right)=\left(\begin{array}{l}
0 \\
0
\end{array}\right)
$$

where $x=p_{0}-m, y=p_{0}+m$, and $\hat{\alpha}, \hat{\delta}, \hat{\epsilon}$ are quaternions with no real part. The relationship between the quaternionic parameters and the SME Lorentz violating parameters can be made explicit. Let $\epsilon^{i j k}$ be the totally antisymmetric symbol on three letters and set

$$
\begin{aligned}
\hat{d}_{1}^{i}=d^{0 i} & \hat{d}_{p}^{i}=d^{i j} p_{j} \\
\hat{H}^{i}=H^{0 i} & \hat{G}^{i}=g^{0 i j} p_{j} \\
\hat{h}^{i}=1 / 2 \epsilon^{i j k} H^{j k} & \hat{g}^{i}=1 / 2 \epsilon^{i j k} g^{j k l} p_{l} .
\end{aligned}
$$

Then

$$
\begin{aligned}
\alpha_{0}=b^{0}+\hat{d}_{1} \cdot \hat{p} & \hat{\alpha}=\hat{H}-\hat{G} \\
\hat{\epsilon}=\hat{b}+\hat{d}_{p}+(\hat{g}-\hat{h}) & \hat{\delta}=-\hat{b}-\hat{d}_{p}+(\hat{g}-\hat{h}) .
\end{aligned}
$$

Note that $a^{\mu}, e^{\mu}$, and $c^{\mu \nu}$ do not appear in the above expressions. These parameters can in fact all be absorbed into a redefined set of momenta and mass parameters that are simply related to the physical momenta and masses.

$$
\begin{aligned}
\hat{p}^{\prime} & =\hat{p}-\hat{a}-\hat{c}_{p} \\
p_{0}^{\prime} & =\left(1+c^{00}\right) p_{0}-a_{0}-\vec{c}_{1} \cdot \vec{p} \\
m^{\prime} & =m-\vec{e} \cdot \vec{p},
\end{aligned}
$$

where $\left(c_{p}\right)^{i}=c^{i j} p^{j}$ and $\left(c_{1}\right)^{i}=c^{0 i}$. The primes on these parameters are dropped from the following calculation for notational convenience since they do not change 
the general quaternionic structure of the equations. In addition, several of these parameters may be removed using appropriate field redefinitions and are therefore not physical anyway [13]. At the end of the calculation, these parameters may be easily included by inverting the above linear equations and solving for the physical momenta and mass parameters. Note that the results of [14] and [15] have been used to remove the $f_{\mu}$ dependence and to arrange for $\Gamma^{0}=\gamma^{0}$. These reductions greatly simplify the computations to follow.

We can use (9)-(12) to compute the effect of $\mathcal{C}, \mathcal{P}$, and $\mathcal{T}$ on the Dirac equation in our representation. For charge conjugation we have

$$
\mathcal{C}:\left(\begin{array}{cc}
x & \alpha_{0}+\hat{\alpha} \\
-\alpha_{0}+\hat{\alpha} & -y
\end{array}\right)+i\left(\begin{array}{cc}
\hat{\epsilon} & \hat{p} \\
-\hat{p} & \hat{\delta}
\end{array}\right) \longrightarrow\left(\begin{array}{cc}
-y & \alpha_{0}+-\hat{\alpha} \\
-\alpha_{0}-\hat{\alpha} & x
\end{array}\right)+i\left(\begin{array}{cc}
-\hat{\delta} & -\hat{p} \\
\hat{p} & -\hat{\epsilon}
\end{array}\right)
$$

while for parity we have

$$
\mathcal{P}:\left(\begin{array}{cc}
x & \alpha_{0}+\hat{\alpha} \\
-\alpha_{0}+\hat{\alpha} & -y
\end{array}\right)+i\left(\begin{array}{cc}
\hat{\epsilon} & \hat{p} \\
-\hat{p} & \hat{\delta}
\end{array}\right) \longrightarrow\left(\begin{array}{cc}
x & -\alpha_{0}-\hat{\alpha} \\
\alpha_{0}-\hat{\alpha} & -y
\end{array}\right)+i\left(\begin{array}{cc}
\hat{\epsilon} & -\hat{p} \\
\hat{p} & -\hat{\delta}
\end{array}\right) .
$$

Using the matrix identification $\gamma^{5} C=-\hat{j} I$, we can compute the effect of time reversal:

$$
\mathcal{T}:\left(\begin{array}{cc}
x & \alpha_{0}+\hat{\alpha} \\
-\alpha_{0}+\hat{\alpha} & -y
\end{array}\right)+i\left(\begin{array}{cc}
\hat{\epsilon} & \hat{p} \\
-\hat{p} & \hat{\delta}
\end{array}\right) \longrightarrow\left(\begin{array}{cc}
x & \alpha_{0}+\hat{\alpha} \\
-\alpha_{0}+\hat{\alpha} & -y
\end{array}\right)+i\left(\begin{array}{cc}
-\hat{\epsilon} & -\hat{p} \\
\hat{p} & -\hat{\delta}
\end{array}\right) .
$$

Using the quaternionic representation of the Dirac equation (19), we can produce a useful expression for the dispersion relation. To do so, solve for the spinor $\phi$ in terms of $\xi$ to obtain

$$
\left[\left(\alpha_{0}-\hat{\alpha}+i \hat{p}\right)(x-i \hat{\epsilon})\left(\alpha_{0}+\hat{\alpha}+i \hat{p}\right)+r(-y+i \hat{\delta})\right] \xi=0
$$

where $r=x^{2}-|\hat{\epsilon}|^{2}$. We write this as

$$
\left(q_{1}+i \hat{q}_{2}\right) \xi=0
$$

where

$$
\begin{aligned}
& q_{1}=x\left(\alpha_{0}^{2}+\vec{\alpha}^{2}+\vec{p}^{2}\right)-2 \alpha_{0} \vec{\epsilon} \cdot \vec{p}-2 \vec{\alpha} \cdot(\vec{\epsilon} \times \vec{p})-r y \\
& \hat{q}_{2}=2 x\left[\alpha_{0} \hat{p}-(p \hat{\times} \alpha)\right]-2 \alpha_{0}(\alpha \hat{\times} \epsilon)-2(\vec{\alpha} \cdot \vec{\epsilon}) \hat{\alpha}-2(\vec{p} \cdot \vec{\epsilon}) \hat{p}+\left(\vec{\alpha}^{2}-\alpha_{0}^{2}+\vec{p}^{2}\right) \hat{\epsilon}+r \hat{\delta}
\end{aligned}
$$


where $v \hat{\times} u$ represents the pure imaginary quaternion obtained by forming the cross product of the two vectors involved and mapping it to the coresponding quaternion.

The analysis of (29) requires the characterization of the null space of $A+i B$ where $A$ and $B$ are quaternionic, with $A=a I, a$ real. It is easy to see that such operators have null space if and only if $B$ has no real part (ie $B$ is traceless) and $\operatorname{det}(A)=\operatorname{det}(B)$. This latter condition can be expressed in terms of the quaternionic $\operatorname{norm}: \operatorname{det}(A)=\operatorname{det}(B)$ if and only if $|A|^{2}=|B|^{2}$.

Returning to (29), note that $q_{1}$ is real and that $\hat{q}_{2}$ is a pure imaginary quaternion. We interpret $i \hat{q}_{2}$ as a linear operator on spinors and (29) as an eigenvalue equation for the relevant operator $i \hat{q}_{2}$. By the above discussion concerning null spaces for quaternionic matrices, the associated polynomial whose roots determine the desired eigenvalues is characterized by the equality $\left|\hat{q}_{2}\right|^{2}=q_{1}^{2}$. Explicit calculation of both sides of this equation yield the dispersion relation as given by the polynomial identity

$$
\sum_{j=0}^{4} \beta_{j} p_{0}^{j}=0
$$

where the coefficients satisfy $\beta_{4}=1, \beta_{3}=0$, and

$$
\begin{aligned}
\beta_{2}= & -2\left[\alpha_{0}^{2}+\vec{\alpha}^{2}+m^{2}+\vec{p}^{2}\right]-\left(\vec{\epsilon}^{2}+\vec{\delta}^{2}\right) \\
\beta_{1}= & 2 m\left(\vec{\delta}^{2}-\vec{\epsilon}^{2}\right)-4 \alpha_{0}(\vec{p} \cdot(\vec{\delta}-\vec{\epsilon}))-4(\vec{\alpha} \times \vec{p}) \cdot(\vec{\epsilon}+\vec{\delta}) \\
\beta_{0}= & \vec{p}^{4}-2 \vec{p}^{2}\left(\alpha_{0}^{2}+\vec{\alpha}^{2}-m^{2}+\vec{\epsilon} \cdot \vec{\delta}\right)+\left(\alpha_{0}^{2}+\vec{\alpha}^{2}+m^{2}\right)^{2}-m^{2}\left(\vec{\delta}^{2}+\vec{\epsilon}^{2}\right)+2(\vec{\epsilon} \cdot \vec{\delta})\left(\alpha_{0}^{2}-\vec{\alpha}^{2}\right) \\
& +\vec{\epsilon}^{2} \vec{\delta}^{2}+4 m\left[(\vec{\alpha} \times \vec{p}) \cdot(\vec{\delta}-\vec{\epsilon})+\alpha_{0} \vec{p} \cdot(\vec{\delta}+\vec{\epsilon})\right]+4 \alpha_{0} \vec{\epsilon} \cdot(\vec{\delta} \times \vec{\alpha}) \\
& +4\left[(\vec{\alpha} \cdot \vec{p})^{2}+(\vec{\epsilon} \cdot \vec{p})(\vec{p} \cdot \vec{\delta})+(\vec{\alpha} \cdot \vec{\delta})(\vec{\epsilon} \cdot \vec{\alpha})\right]
\end{aligned}
$$

The cubic term of the above fourth order monic polynomial vanishes, therefore the polynomial admits a generic factorization of the form

$$
\left(p_{0}^{2}+t p_{0}+u\right)\left(p_{0}^{2}-t p_{0}+v\right)
$$

where $t, u$, and $v$ are parameters that depend on the vector momentum as well as the Lorentz-violating parameters, but in general their explicit expressions are cumbersome and not particularly insightful. Note that when the linear term also vanishes, the 
complete factorization is particularly simple. We pursue this and other consequences of the dispersion formula (30) in the next section.

\section{SPECIAL CASES OF DISPERSION FORMULA}

When the Lorentz violating parameters are set to zero, the dispersion relation factors into the square of a second-order polynomial

$$
\left(p_{0}^{2}-\vec{p}^{2}-m^{2}\right)^{2}=0
$$

Fixing the value of $p_{0}$ defines a doubly degenerate sphere in momentum space. Thus, when the Lorentz violating parameters are small with respect to mass and momentum, the dispersion relation is expected to define a smoothly perturbed sphere with possible multiple sheets. We show below that the resulting surface is generically a doublesheeted perturbation of the sphere.

We begin by noticing that the dispersion relation becomes particularly simple to analyze when the coefficient of the linear term $\beta_{1}$ given in (32) vanishes. In order for this to occur for arbitrary $\vec{p}$ we must have either $\vec{\epsilon}+\vec{\delta}=0$ or $\vec{\epsilon}-\vec{\delta}=0$. We analyze each case separately.

Case: $\vec{\epsilon}+\vec{\delta}=0$

When $\vec{\epsilon}=-\vec{\delta}$, to ensure that $\beta_{1}=0$ for arbitrary $\vec{p}$, it is sufficient that $\alpha_{0}=0$. In this case, the coefficients of the polynomial (30) become

$$
\begin{aligned}
\beta_{2} & =-2\left(\vec{p}^{2}+m^{2}+\vec{\alpha}^{2}+\vec{\delta}^{2}\right) \\
\beta_{0} & =\left(\vec{p}^{2}+m^{2}-\vec{\alpha}^{2}+\vec{\delta}^{2}\right)^{2} \\
& \left.+4\left[\vec{\delta}^{2} \vec{\alpha}^{2}-m^{2} \vec{\delta}^{2}+m^{2} \vec{\alpha}^{2}+(\vec{\alpha} \cdot \vec{p})^{2}-(\vec{\delta} \cdot \vec{p})^{2}-(\vec{\alpha} \cdot \vec{\delta})^{2}+2 m(\vec{\alpha} \times \vec{p}) \cdot \vec{\delta}\right)\right] .
\end{aligned}
$$

When this is the case, completing the square in the associated dispersion relation yields

$$
p_{0}^{2}=\vec{p}^{2}+m^{2}+\vec{\alpha}^{2}+\vec{\delta}^{2} \pm 2 \sqrt{D_{1}(\vec{p})}
$$

where

$$
D_{1}(\vec{p})=(\vec{\alpha} \times \vec{p}-m \vec{\delta})^{2}+(\vec{\delta} \cdot \vec{p})^{2}+(\vec{\alpha} \cdot \vec{\delta})^{2}
$$


is a non-negative quantity, as is required for reality of the energy eigenvalues.

Case: $\vec{\epsilon}-\vec{\delta}=0$

When $\vec{\epsilon}-\vec{\delta}=0$, to ensure that $\beta_{1}=0$ for arbitrary $\vec{p}$, it is sufficient that $\vec{\alpha}=0$. The coefficients of the polynomial (30) become

$$
\begin{aligned}
& \beta_{2}=-2\left(\vec{p}^{2}+m^{2}+\alpha_{0}^{2}+\vec{\delta}^{2}\right) \\
& \beta_{0}=\left(\vec{p}^{2}+m^{2}-\alpha_{0}^{2}-\vec{\delta}^{2}\right)^{2}+4 m^{2} \alpha_{0}^{2}+8 m \alpha_{0} \vec{p} \cdot \vec{\delta}+4(\vec{p} \cdot \vec{\delta})^{2} .
\end{aligned}
$$

Completing the square yields the solutions

$$
p_{0}^{2}=\vec{p}^{2}+m^{2}+\alpha_{0}^{2}+\vec{\delta}^{2} \pm 2 \sqrt{D_{2}(\vec{p})}
$$

where

$$
D_{2}(\vec{p})=(\vec{\delta} \times \vec{p})^{2}+\left(\alpha_{0} \vec{p}-m \vec{\delta}\right)^{2} .
$$

Note that $D_{2}(\vec{p}) \geq 0$ as in the first case.

In both of these special cases, the dispersion relation is symmetric under $p_{0} \rightarrow-p_{0}$ indicating that positive and (reinterpreted) negative energy states are degenerate. In addition, for a fixed value of $p_{0}$, the set of solutions for $\vec{p}$ forms a deformed sphere with two sheets where the radius as a function of angle is determined by the relevant factor, $D_{1}(\vec{p})$, or $D_{2}(\vec{p})$. This simple geometric interpretation works well provided that the Lorentz-violation parameters are small relative to the momentum and mass involved. Special degeneracies may arise when the Lorentz-violating parameters become comparable to the size of the momentum or mass involved.

Recalling the relationship between the Lorentz violating parameters and the quaternionic parameters (20)-(21), we see that the quaternionic representation determines mutually exclusive special cases for which the analysis of the dispersion relation is easy. These special cases partition the Lorentz violating parameters. The case $\vec{\epsilon}=\vec{\delta}$, with corresponding condition $\vec{\alpha}=0$ corresponds to setting $\hat{b}-\hat{d}_{p}=0$ and $\hat{H}-\hat{G}=0$, implying that $\hat{b}=\hat{d}_{p}=\hat{H}=\hat{G}=0$ since the relations must hold for arbitrary values of the momentum $\vec{p}$. The other Lorentz-violation parameters may be left arbitrary. The case $\vec{\epsilon}=-\vec{\delta}$, with corresponding condition $\alpha_{0}=0$ corresponds to setting $\hat{g}-\hat{h}=0$ and $b^{0}+\hat{d}_{1} \cdot \hat{p}=0$, while leaving the other parameters arbitrary. Again, 
this condition implies $\hat{g}=\hat{h}=b_{0}=\hat{d}_{1}=0$ all vanish, while the other parameters remain arbitrary.

When the linear term in (30) does not vanish, it is sometimes still possible to give a complete analysis of the dispersion relation. We consider two special cases of particular interest: $b^{\mu}$, and $H^{\mu \nu}$.

Case: $b^{\mu} \neq 0$, or $\hat{\epsilon}=-\hat{\delta}$, but with $\alpha_{0} \neq 0$ :

This case corresponds to the special case where the only nonvanishing Lorentz violating parameters are taken to be the vector $b^{\mu}$. Using the expression (30), the dispersion relation becomes

$$
\left(p_{0}^{2}-\vec{p}^{2}-m^{2}-b_{0}^{2}-\vec{b}^{2}\right)^{2}-4\left(b_{0}^{2} \vec{p}^{2}+b_{0}^{2} \vec{b}^{2}+(\vec{b} \cdot \vec{p})^{2}+m^{2} \vec{b}^{2}\right)+8 b_{0} p_{0}(\vec{b} \cdot \vec{p})=0
$$

Direct solution is difficult due to the nonvanishing linear coefficient in $p_{0}$, however, we can still make some progress in obtaining the general structure of the solution space by writing $\vec{b}=|\vec{b}| \hat{v}$ and decomposing $\vec{p}$ accordingly: $\vec{p}=t \hat{v}+\vec{z}$, where $\vec{z}$ is the component of momentum perpendicular to $\hat{v}$. Then

$$
\left(\vec{z}^{2}-\left(p_{0}^{2}-m^{2}+b_{0}^{2}-\vec{b}^{2}-t^{2}\right)\right)^{2}=4\left(\left(|\vec{b}| t-p_{0} b_{0}\right)^{2}+m^{2}\left(\vec{b}^{2}-b_{0}^{2}\right)\right) .
$$

To solve for $|\vec{z}|$ as a function of $t$, set

$$
\begin{aligned}
& f_{1}(t)=p_{0}^{2}-m^{2}+b_{0}^{2}-\vec{b}^{2}-t^{2} \\
& f_{2}(t)=4\left(\left(|\vec{b}| t-p_{0} b_{0}\right)^{2}+m^{2}\left(|\vec{b}|^{2}-b_{0}^{2}\right)\right)
\end{aligned}
$$

Note that for a solution to exist we must have $f_{2}(t) \geq 0$ and $f_{1}(t) \geq-\sqrt{f_{2}(t)}$. To proceed, assume $r^{2}=b_{0}^{2}-\vec{b}^{2}>0$. Set

$$
\begin{aligned}
& x_{0}=\frac{1}{r}\left(b_{0} p_{0}-|\vec{b}| t\right) \\
& x_{1}=\frac{1}{r}\left(-|\vec{b}| p_{0}+b_{0} t\right)
\end{aligned}
$$

Then

$$
\begin{aligned}
& f_{1}(t)=x_{0}^{2}-x_{1}^{2}-m^{2}+r^{2} \\
& f_{2}(t)=4 r^{2}\left(x_{0}^{2}-m^{2}\right)
\end{aligned}
$$


and $f_{1}^{2}-f_{2}$ factors:

$$
f_{1}^{2}-f_{2}=\left(\left(x_{1}+r\right)^{2}-\left(x_{0}^{2}-m^{2}\right)\right)\left(\left(x_{1}-r\right)^{2}-\left(x_{0}^{2}-m^{2}\right)\right)
$$

When $\vec{z}=0$, the momentum points purely in the $\vec{b}$ direction and $f_{1}^{2}-f_{2}=0$ giving the endpoint conditions on the momentum:

$$
\begin{aligned}
& \left(t+b_{0}\right)^{2}=\left(p_{0}+|\vec{b}|\right)^{2}-m^{2} \\
& \left(t-b_{0}\right)^{2}=\left(p_{0}-|\vec{b}|\right)^{2}-m^{2}
\end{aligned}
$$

A similar analysis for the case $r=\vec{b}^{2}-b_{0}{ }^{2}>0$ leads to the same pair of equations. and thus, we generically obtain the four solutions:

$$
\begin{aligned}
t & =-b_{0} \pm \sqrt{\left(p_{0}+|\vec{b}|\right)^{2}-m^{2}} \\
t & =b_{0} \pm \sqrt{\left(p_{0}-|\vec{b}|\right)^{2}-m^{2}}
\end{aligned}
$$

Case: $H^{\mu \nu} \neq 0$, or $\hat{\epsilon}=\hat{\delta}$, but with $\hat{\alpha} \neq 0$ :

This case corresponds to the special case where the only nonvanishing Lorentz violating parameters are taken to be the tensor $H^{\mu \nu}$. Using the expression (20)-(21), the coefficients in the dispersion relation become

$$
\begin{aligned}
\beta_{2}= & -2\left[\vec{H}^{2}+m^{2}+\vec{p}^{2}+\vec{h}^{2}\right] \\
\beta_{1}= & 8(\vec{H} \times \vec{p}) \cdot \vec{h} \\
\beta_{0}= & \vec{p}^{4}-2 \vec{p}^{2}\left(\vec{H}^{2}-m^{2}+\vec{h}^{2}\right)+\left(\vec{H}^{2}+m^{2}\right)^{2}-2 m^{2} \vec{h}^{2}-2 \vec{h}^{2} \vec{H}^{2}+ \\
& \vec{h}^{4}+4\left[(\vec{H} \cdot \vec{p})^{2}+(\vec{h} \cdot \vec{p})^{2}+(\vec{H} \cdot \vec{h})^{2}\right] .
\end{aligned}
$$

To analyze the corresponding dispersion relation we begin by assuming that $\vec{H}$ and $\vec{h}$ are colinear: $\vec{H}=s \vec{h}$. Then

$$
\begin{aligned}
\beta_{2}= & -2\left[m^{2}+\vec{p}^{2}+\left(1+s^{2}\right) \vec{h}^{2}\right] \\
\beta_{1}= & 0 \\
\beta_{0}= & \vec{p}^{4}-2 \vec{p}^{2}\left(\left(1+s^{2}\right) \vec{h}^{2}-m^{2}\right)+\left(s^{2} \vec{h}^{2}+m^{2}\right)^{2}-2 m^{2} \vec{h}^{2}-2 s^{2} \vec{h}^{4}+ \\
& \vec{h}^{4}+4\left[\left(1+s^{2}\right)(\vec{h} \cdot \vec{p})^{2}+s^{2} \vec{h}^{4}\right] .
\end{aligned}
$$


Completing the square, we obtain the solutions in a familiar form

$$
p_{0}^{2}=\vec{p}^{2}+m^{2}+\left(1+s^{2}\right) \vec{h}^{2} \pm 2 \sqrt{D_{3}(\vec{p})},
$$

where

$$
D_{3}(\vec{p})=\left(1+s^{2}\right)(\vec{h} \times \vec{p})^{2}+m^{2} \vec{h}^{2} .
$$

Note that the variety (for fixed $p_{0}$ ) takes the form of two nested, pertubed spheres in momentum space, as expected.

When $\vec{h}$ and $\vec{H}$ are not parallel, we can apply an observer Lorentz boost to reduce to the parallel case. To proceed, note that the components of $\vec{h}$ and $\vec{H}$ define the components of the antisymmetric 2-tensor appearing in the Lagrangian (3)-(5) via the expression

$$
H^{\mu \nu}=\left(\begin{array}{cccc}
0 & H^{1} & H^{2} & H^{3} \\
-H^{1} & 0 & h_{3} & -h_{2} \\
-H^{2} & -h_{3} & 0 & h_{1} \\
-H^{3} & h_{2} & -h_{1} & 0
\end{array}\right)
$$

In particular, the triple $\left(H^{\mu \nu}, \vec{H}, \vec{h}\right)$ defines an object which can be analyzed using the techniques used to treat the triple $\left(F^{\mu \nu}, \vec{E}, \vec{B}\right)$ where $F^{\mu \nu}$ is electromagnetic field strength, $\vec{E}$ is a (static) electric field and $\vec{B}$ is a (static) magnetic field. Assuming that $|\vec{h}|>|\vec{H}|$, write $\vec{H}=\vec{H}_{\|}+\vec{H}_{\perp}$ where $\vec{H}_{\|}$is the component of $\vec{H}$ in the direction of $\vec{h}$ and $\vec{H}_{\perp}$ is perpendicular to $\vec{h}$. Consider the boost

$$
\vec{u}=\frac{\vec{H}_{\perp} \times \vec{h}}{\vec{h}^{2}}
$$

and set $\gamma=\left(1-\vec{H}_{\perp}^{2} / \vec{h}^{2}\right)^{-\frac{1}{2}}$. Then, by direct calculation, in the coordinates associated to the boosted frame $\vec{u}$ the vectors $\vec{H}$ and $\vec{h}$ are given by

$$
\begin{aligned}
\vec{H}^{\prime} & =\gamma \vec{H}_{\|} \\
\vec{h}^{\prime} & =\gamma^{-1} \vec{h} .
\end{aligned}
$$

A similar argument applies when $|\vec{h}|<|\vec{H}|$ by interchanging the roles of the vectors and the analysis is reduced to the previous special case in the new frame.

\section{SUMMARY}

In this paper we have provided a detailed investigation of the dispersion relation associated to the Dirac operator for the SME using an approach which employed 
quaternions. The traditional $4 \times 4$ structure of the Dirac equation can be re-expressed in $2 \times 2$ form where the entries are quaternion valued. This greatly simplifies the matrix structure at the expense of loss of commutativity of the matrix elements. While it is possible to perform all of the computations using the traditional $4 \times 4$ notation, the quaternion valued elements provide an efficient way to organize the computation.

Generically, our results exhibit the dispersion relation as a pair of perturbed spheres. We find that the dispersion relation can be easily solved for two special sets of parameter choices. Each choice allows half of the Lorentz violating parameter space to be "turned on" while the other half of the space is "turned off". This provides a practical way to approach analysis involving the fermions in the SME with each special case providing simple insight into the effect of the relevant terms. Many problems that are intractable using a general fourth-order dispersion relation become far simpler to analyze when the dispersion relation factors into two second-order ones. In particular, the analysis of the dispersion relation for a theory involving Lorentz violation coupled to spin through the $H^{\mu \nu}$ term in the Lagrangian defined by (3)-(5) can be carried out explicitly. In addition to the above results, further exploitation of the representation (for example, the use of the dispersion relation to label eigenstates) provide interesting directions for probing hidden symmetries of the SME.

ACKNOWLEDGMENTS We wish to acknowledge the support of New College of Florida's faculty development funds that contributed to the successful completion of this project.

\section{REFERENCES}

1. V.A. Kostelecký and S. Samuel, Phys. Rev. D 39, 683 (1989); ibid. 40, 1886 (1989); Phys. Rev. Lett. 63, 224 (1989); ibid. 66, 1811 (1991); V.A. Kostelecký and R. Potting, Nucl. Phys. B 359, 545 (1991); Phys. Lett. B 381, 89 (1996); Phys. Rev. D 63, 046007 (2001); V.A. Kostelecký, M. Perry, and R. Potting, Phys. Rev. Lett. 84, 4541 (2000). 
2. D. Colladay and V.A. Kostelecký, Phys. Rev. D 55, 6760 (1997); Phys. Rev. D 58, 116002 (1998).

3. For a summary of recent theoretical models and experimental tests see, for example, CPT and Lorentz Symmetry III, V.A. Kostelecký, ed., World Scientific, Singapore, 2005; CPT and Lorentz Symmetry IV, V.A. Kostelecký, ed., World Scientific, Singapore, 2008.

4. V.A. Kostelecký and R. Lehnert, Phys. Rev. D 63, 065008 (2001).

5. V.A. Kostelecký, C. Lane, and A. Pickering, Phys. Rev. D 65, 056006 (2002).

6. V.A. Kostelecký and A. Pickering, Phys. Rev. Lett. 91, 031801 (2003).

7. D. Colladay and P. McDonald, Phys. Rev. D 75, 105002 (2007); Phys. Rev. D 77, 085006 (2008); Phys. Rev. D 79, 125019 (2009).

8. A. Kostelecký and R. Potting, Phys Rev. D 79, 065018 (2009).

9. A. Kostelecky and M. Mewes, Phys. Rev. Lett. 99, 011601 (2007).

10. V. A. Kostelecký and N. Russell arXiv:0801.0287

11. R. Lehnert, J. Math. Phys. 453399 (2004).

12. See, for example, D. Schuricht and M. Greiter Eur. J. Phys. 25755 (2004).

13. D. Colladay and P. McDonald, J. Math. Phys. 433554 (2002).

14. B. Altschul, J. Phys. A 39,13757 (2006).

15. R. Lehnert, Phys. Rev. D 63, 065008 (2001). 